Takasumi Katoh MD, Yoshitada Suguro MD, Takeshi Kimura MD, Kazuyuki Ikeda MD

\title{
Morphine does not affect the awakening concentration of sevoflurane
}

This study was designed to determine whether morphine 0.1 $m \mathrm{~g} \cdot \mathrm{kg}^{-1}$ iv given intraoperatively altered the end-tidal concentration of sevoflurane which is associated with eye opening 10 verbal command. We studied 24 healthy ASA physical status I patients to determine whether morphine, or placebo administered about $60 \mathrm{~min}$ before the end of surgery affected recovery from sevoflurane/oxygen anaesthesia. During anaesthesia no other anaesthetics or drugs were given. Afier surgery, end-tidal sevoflurane concentration was reduced gradually at the rate of less than $0.01 \% \cdot \mathrm{min}^{-1}$. The end-tidal concentration at the time patients could respond to verbal command was recorded as MACawake. The MACawake was $0.58 \pm 0.12 \%$ (mean \pm $S D)$ for the control group to whom placebo had been administered, and $0.57 \pm 0.11 \%$ for morphine group to whom morphine had been administered. In both groups, the MACawake decreased with age, and the ratio to age-adjusted sevoflurane $M A C$ was $0.31 \pm 0.04$ (mean $\pm S D$ ) for the control group and $0.30 \pm 0.04$ for the morphine group. The ratio had no correlation with age. It is concluded that the awakening concentration of sevoflurane during recovery from anaesthesia is not affected by analgesic doses of morphine $0.1 \mathrm{mg} \cdot \mathrm{kg}^{-1}$ iv administered intraoperatively.

Cette étude a pour but de savoir si la morphine, $0,1 \mathrm{mg} \cdot \mathrm{kg}^{-1}$ $\dot{N}$ donnée en peropératoire altère la concentration de fin d'expiration du sévoflurane correspondant à l'ouverture des yeux à la commande verbale. Nous avons étudié 24 patients sains ASA I pour déterminer si la morphine ou un placebo administré environ $60 \mathrm{~min}$ avant la fin de lintervention affecte le réveil d'une anesthésie au sévoflurane et oxygène. Pendant

\section{Key words}

ANAESTHETICS, VOLATILE: sevoflurane; POTENCY: MAC.

From the Department of Anesthesiology and Intensive Care, Hamamatsu University School Medicine.

Address correspondence 10: Dr. Takasumi Katoh, Department of Anesthesiology and Intensive Care,

Hamamatsu University School of Medicine, 3600 Handa-cho,

Hamamatsu, 431-31 Japan.

Accepted for publication 21st May, 1993. l'anesthésie, aucun autre agent d'anesthésie ou médicament n'ont été administrés. Après l'intervention, la concentration de fin d'expiration du sévoflurane a été graduellement réduite à une vitesse de moins de $0,01 \%$ par minute. La concentration de fin d'expiration au moment où les patients pouvaient répondre à la commande verbale a été enregistrée comme CAM d'éveil. Le CAM d'éveil est de 0,58 $\pm 0,12 \%$ (moyenne \pm écart type) pour le groupe contrôle qui a reçu le placebo et de 0,57 $\pm 0,11 \%$ pour le groupe auquel la morphine a été administrée. Dans les deux groupes, le CAM d'éveil diminue avec l'âge et la valeur du CAM du sévoflurane corrigée selon l'âge est de $0,31 \pm 0,04$ (moyenne \pm écart type) pour le group contrôle et de 0,30 $\pm 0,04$ pour le groupe morphine. Il n'y a pas de corrélation avec l'áge. On conclut que la concentration d'éveil du sévoflurane au réveil de l'anesthésie n'est pas affectée par des doses analgésiques de morphine $0,1 \mathrm{mg} \cdot \mathrm{kg}^{-1}$ iv administrées en peropératoire.

Sevoflurane has a low blood-gas partition coefficient, and patients awake rapidly from sevoflurane anaesthesia. Many anaesthetists have experienced patients in the recovery room who had suffered pain after very rapid and clear awakening from sevoflurane anaesthesia. To relieve the pain, analgesics may be administered intraoperatively, but this may delay rapid recovery from anaesthesia which is one of the advantages of sevoflurane. This study was designed to determine whether morphine 0.1 $\mathrm{mg} \cdot \mathrm{kg}^{-1} i \mathrm{v}$ given intraoperatively altered the end-tidal concentration of sevoflurane which is associated with eye opening to verbal command.

\section{Methods}

With local ethics committee approval and informed patient consent, we studied 24 patients of either sex, all of ASA physical status I, and who were scheduled for elective extremity, or surface surgery. Patients were excluded if they received another kind of surgery, or if a laryngeal mask airway was contraindicated. Patients were monitored in the routine fashion. They fasted for at least eight hours before surgery and received no premedicant drugs. Anaesthesia was induced with sevoflu- 
TABLE Summary of results

\begin{tabular}{lcc}
\hline & Placebo & Morphine \\
\hline$n$ & 12 & 12 \\
Age (yr) & $45.3 \pm 16.5$ & $43.3 \pm 15.7$ \\
Weight (kg) & $52 \pm 10$ & $56 \pm 10$ \\
$\begin{array}{l}\text { Total duration of sevoflurane } \\
\quad \text { administration (min) }\end{array}$ & $140 \pm 21$ & $161 \pm 51$ \\
$\begin{array}{l}\text { Time from morphine or placebo } \\
\quad \text { until sevoflurane wears off }\end{array}$ & $53 \pm 10$ & $62 \pm 11$ \\
MACawake (\%) & $0.58 \pm 0.12$ & $0.57 \pm 0.11$ \\
MACawake:MAC ratio & $0.31 \pm 0.04$ & $0.30 \pm 0.04$ \\
Respiratory rate & $14.0 \pm 1.4$ & $13 \pm 1.3$ \\
PaCO $_{2}$ & $40.6 \pm 5.3$ & $44.6 \pm 4.3$ \\
\hline
\end{tabular}

Values are means $\pm \mathrm{SD}$.

rane and oxygen during spontaneous ventilation. The Brain laryngeal mask airway was inserted without the use of muscle relaxants or other drugs. After that, we confirmed that no gas leaked from the margin of the cuff of the laryngeal mask airway when the lungs were ventilated with positive airway pressure of about 20 $\mathrm{cmH}_{2} \mathrm{O}$ and that the end-tidal concentration of nitrogen was $<1 \%$. Throughout surgery, anaesthesia was maintained with $50 \%$ oxygen, sevoflurane and balanced nitrogen but with no other drugs. Mechanical ventilation was performed to keep end-tidal carbon dioxide concentration from 33 to $40 \mathrm{mmHg}$. The end-tidal concentrations of sevoflurane and carbon dioxide were measured continuously by means of an infrared multigas anaesthetic gas analyzer (Capnomac Ultima, Datex, Finland). Gas samples were collected with a Teflon catheter placed at the laryngeal end of the laryngeal mask at the rate of $200 \mathrm{ml} \cdot \mathrm{min}^{-1}$. The concentration of nitrogen was measured intermittently every minute by means of a mass spectrometer (MGA-1100, Perkin Elmer, Ca). The anaesthetic concentration was varied to facilitate surgery. Approximately one hour before the end of surgery, patients received, by random allocation, either morphine 0.1 $\mathrm{mg} \cdot \mathrm{kg}^{-1}$ (morphine group) or an equal volume of saline placebo $i v$ (control group). Anesthetists and an observer were blinded to which was given to a patient, placebo or morphine.

Following surgery, anaesthesia was discontinued. When spontaneous ventilation was stable, the laryngeal mask airway was removed, and $100 \%$ oxygen was administered from a mask held above the face. After the end-expired anaesthetic concentration, which was sampled $7 \mathrm{~cm}$ inside the nasal cavity, decreased to $1.2 \%$, a low concentration of anaesthetic was added to oxygen to prevent the end-tidal anaesthetic concentration from decreasing too rapidly. We kept the rate of decline of end-tidal anaesthetic concentration at less than

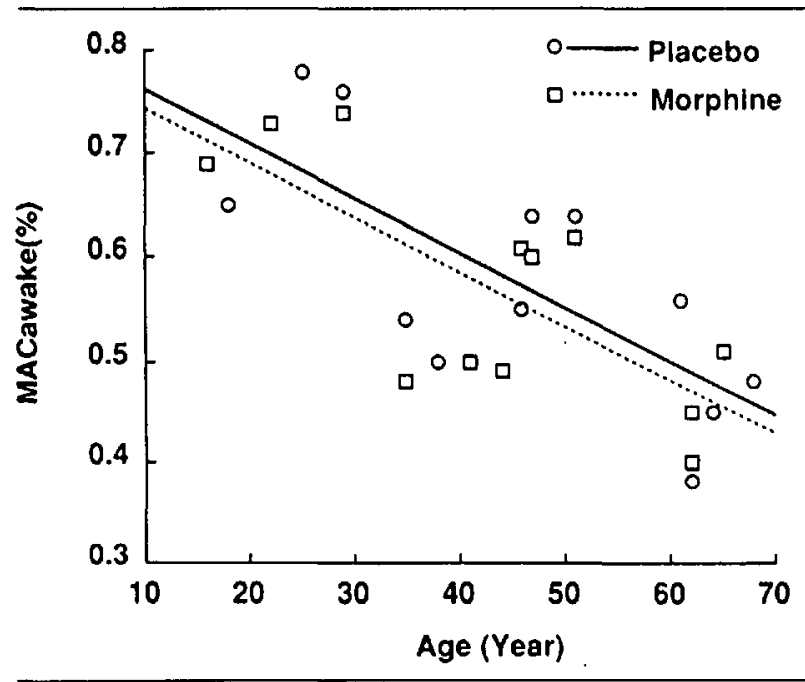

FIGURE Correlation between age and end-tidal concentration of sevoflurane on awakening in both of control and morphine groups.

$0.01 \% \cdot \mathrm{min}^{-1}$. Patients were asked at frequent intervals to open their eyes. To avoid acclimatizing the patients to the aural stimulus, the command was repeated for 20 sec every minute. The concentration when the patient first opened his eyes in response to command was defined as MACawake. Respiratory rate and $\mathrm{PaCO}_{2}$ were measured in the recovery room.

To compensate for the effect of age on anaesthetic requirements, ${ }^{1-4}$ we computed the ratio of MACawake to the age-adjusted sevoflurane MAC for each patient. Oneway analysis of variance was used to compare variables between groups; correlation analysis was used to determine if age affected MACawake. A $P$ value $<0.05$ was considered significant. A power analysis was performed to indicate the power of the study for detecting differences in MACawake and the ratio of MACawake to ageadjusted $\mathrm{MAC}$.

\section{Results}

Patient ages, total duration of sevoflurane administration, time from administration of morphine or placebo until the end of surgery, and respiratory rate and $\mathrm{PaCO}_{2}$ in the recovery room did not differ between groups (Table). For patients receiving morphine MACawake (0.57 土 $0.11 \%$, mean $\pm \mathrm{SD}$ ) did not differ from that in patients receiving placebo $(0.58 \pm 0.11 \%)$.

Awakening concentration correlated with age $(P<$ 0.05 ) in both groups ( $\mathrm{r}=0.73, P<0.01$ for placebo group; $\mathrm{r}=0.74, P<0.01$ for morphine group) (Figure). The awakening concentration:age-adjusted MAC ratio also did not differ between the morphine $(0.30 \pm 0.04$, mean $\pm \mathrm{SD})$ and placebo $(0.31 \pm 0.04)$ groups, and did not correlate with the patients' age. A power analysis revealed that real differences of MACawake of $0.15 \%$, 
or that of MACawake:MAC ratio of 0.05 could have occurred with a probability of $>80 \%$.

\section{Discussion}

Morphine failed to decrease the awakening concentration of sevoflurane. It may be that too much time had elapsed between morphine administration and MACawake determination. However, Pandit et al. showed that $0.5 \mathrm{mg}$ morphine $i v$ provided greater pain relief than placebo for six hours after its administration. ${ }^{5}$ Gross and Alexander have demonstrated that intraoperative administration of morphine did not alter the awakening concentration of isoflurane. ${ }^{6}$ Morphine may provide more analgesia than sedation and after $0.1 \mathrm{mg} \cdot \mathrm{kg}^{-1}$ some patients respond to verbal stimuli despite profound analgesia. ${ }^{7}$ One hour after morphine $0.1 \mathrm{mg} \cdot \mathrm{kg}^{-1} \dot{i}$, sedative or hypnotic effects may not be sufficient to delay awakening. Watch et al. reported that the concentration of halothane at which children opened their eyes spontaneously was not affected by morphine administered intraoperatively. ${ }^{8}$ The present study extends this to sevoflurane anaesthesia in adults.

The finding that sevoflurane MACawake decreased with age agrees with our previous report. The ratio of MACawake to age-adjusted MAC did not correlate with age. This suggests that MACawake decreased at a similar rate as the decrease in MAC. Morphine did not affect the negative correlation between age and MACawake.

Morphine $0.1 \mathrm{mg} \cdot \mathrm{kg}^{-1} \dot{i}$ did not affect $\mathrm{PaCO}_{2}$ and respiratory rate in the recovery room. However, Jordan et al. showed that morphine $10 \mathrm{mg} \cdot 70 \mathrm{~kg}^{-1} i v$ caused respiratory depression assessed with end-tidal carbon dioxide tension and the slope of the ventilatory response over the first $3.5 \mathrm{hr}$ after administration. ${ }^{9}$ Hug et al. found that respiratory depression peaks $60-90 \mathrm{~min}$ after $i v$ morphine. ${ }^{10}$ The relatively small doses of morphine administered in this study may explain that we could not detect increase in $\mathrm{PaCO}_{2}$ and decrease in respiratory rate.

The end-tidal anaesthetic concentration at the first response to verbal command has been defined as MACawake by Stoelting et al. A previous report suggested that the MACawake to MAC ratios were fairly close for methoxyflurane, halothane, ether, and fluroxene, being $0.52,0.52,0.67$, and 0.70 , respectively. ${ }^{11}$ The MACawake was determined in two ways: first using a steady state method, in which the investigators determined responsiveness to verbal commands after holding anaesthetic concentrations constant for $15 \mathrm{~min}$. In the other, spontaneous recovery from anaesthesia was allowed. In these patients, the inspired anaesthetic concentrations were close to zero, and no attempt was made to hold alveolar concentration constant. The MACawake determined with spontaneous recovery may underestimate anaesthetic con- centration in the brain because alveolar anaesthetic concentrations are lower than cerebral tensions during recovery from anaesthesia. The brain-alveolar gradient is great with anaesthetics which have low blood and tissue solubility such as sevoflurane, because alveolar concentration decreases rapidly when their administration ceases. This gradient is so small during recovery from an anaesthetic which has high blood and tissue solubility that MACawake determined with spontaneous recovery does not differ from that of the steady state method.

The MACawake from sevoflurane anaesthesia determined with spontaneous recovery method might underestimate the anaesthetic concentration at the time a patient would open his eyes, because of its small blood-gas partition coefficient, $0.63 .{ }^{12}$ We reported that awakening concentration of sevoflurane determined with steady state method was $0.62 \pm 0.02 \%$ (mean $\pm \mathrm{SE}$ ) in 21 patients aged $42.9 \pm 15.3 \mathrm{yr}$ (mean $\pm \mathrm{SD}$ ). The awakening concentration correlated with age $(P<0.001)$, but not with the duration of anaesthesia, sex, or type of surgery, and it decreased at a similar rate as its decrease in MAC with increasing age. Therefore, the ratios to MAC are fairly constant, being $0.34 .{ }^{13}$ In this study, we used a modified spontaneous recovery method, in which sevoflurane was maintained at low concentration in inspired gas to avoid end-tidal concentration decreasing rapidly. As a result, the awakening concentration was $0.58 \pm$ $0.12 \%$ (mean $\pm \mathrm{SD}$ ), which was not different from the $0.62 \%$ determined with the steady state method.

In summary, we conclude that intraoperative morphine $0.1 \mathrm{mg} \cdot \mathrm{kg}^{-1} i v$ does not affect the MACawake of sevoflurane and the negative correlation between MACawake and age.

\section{References}

1 Gregory GA, Eger EI II, Munson ES. The relationship between age and halothane requirement in man. Anesthesiology 1969; 30: 488-91.

2 Katoh $T$, Ikeda $K$. The minimum alveolar concentration (MAC) of sevoflurane in humans. Anesthesiology 1978; 66: 301-3.

3 Scheller MS, Saidman $L J$, Partrige BL. MAC of sevoflurane in humans and the New Zealand white rabbit. Can J Anaesth 1988; 35: 153-6.

4 Katoh $T$, Ikeda $K$. Minimum alveolar concentration of sevoflurane in children. Br J Anaesth 1992; 68: 139-41.

5 Pandit UA, Kothary SP, Pandit SK. Intravenous dezocine for postoperative pain: a double-blind, placebo-controlled comparison with morphine. J Clin Pharmacol 1986; 26: 275-80.

6 Gross JB, Alexander CM. Awakening concentrations of isoflurane are not affected by analgesic doses of morphine. Anesth Analg 1988; 67: 27-30. 
7 Lowenstein E. Morphine "anesthesia" - a perspective (Editorial). Anesthesiology 1971; 35: 563-5.

8 Watcha $M F$, Lagueruela $R G$, White $P F$. Effect of intraoperative analgesic therapy on end-expired concentrations of halothane associated with spontaneous eye opening in children. Anesth Analg 1991; 72: 190-3.

9 Jordan C, Lehane JR, Robson JG, Jones JG. A comparison of the respiratory effects of meptazinol, pentazocine and morphine. Br J Anaesth 1979; 51: 497-502.

10 Hug CC Jr, Murphy MR, Rigel EP, Olson WA Pharmacokinetics of morphine injected intravenously into the anesthetized dog. Anesthesiology 1981; 54: 38-47.

11 Stoelting RK, Longnecker DE, Eger EI II. Minimum alveolar concentrations in man on awakening from methoxyflurane, halothane, ether and fluroxene anesthesia: MAC awake. Anesthesiology 1970; 33: 5-9.

12 Strum DP, Eger EI II. Partition coefficients for sevoflurane in human blood, saline, and olive oil. Anesth Analg 1987; 66: 654-6.

13 Katoh T, Suguro Y, Ikeda T, Kazema T. Ikeda K. Influence of age on awakening concentrations of sevoflurane and isoflurane. Anesth Analg 1993; 76: 384-52. 